\title{
Misfolded, protease-resistant proteins in animal models and human neurodegenerative disease
}

\author{
Dennis W. Dickson \\ Departments of Pathology (Neuropathology) and Neuroscience, Mayo Clinic, Jacksonville, Florida \\ J. Clin. Invest. 110:1403-1405 (2002). doi:10.1172/JCI200217164.
}

Neurodegeneration in the substantia nigra has been known to be a constant feature of Parkinson disease (PD) since the early part of the twentieth century, when Trétiakoff (1) correlated the clinical findings of patients with PD with postmortem pathology. A few years earlier, Lewy had described concentric hyaline inclusions within neurons, which are now referred to as Lewy bodies (LBs) (2). LBs are a sine qua non for the neurodegeneration that characterizes $\mathrm{PD}$, but they are also found in nonparkinsonian disorders, most notably dementia with Lewy bodies (3). LBs are composed of filamentous aggregates of $\alpha$-synuclein (4), and immunohistochemistry for synuclein is currently the most sensitive method for detecting LBs in brain tissue. Ubiquitin is another key component of LBs (5), and widespread use of immunohistochemical methods for detecting LBs, first with ubiquitin and later with synuclein antibodies, demonstrated that pathology in these disorders extended beyond LBs within neuronal perikarya to fibrillar lesions within neuritic processes, so-called Lewy neurites. Neuritic pathology is more widespread than LBs and affects regions of the brain, such as the hippocampus (6), that were not previously considered affected in PD. More recently, using antibodies to modified forms of $\alpha$-synuclein (7) or sensitive antigen retrieval methods, it has been discovered that LBs are more prevalent and Lewy neu-

\footnotetext{
Address correspondence to: Dennis W. Dickson, Department of Pathology, Mayo Clinic Jacksonville, 4500 San Pablo Road, Jacksonville, Florida 32224, USA. Phone: (904) 953-7137; Fax: (904) 953-7117; E-mail: dickson.dennis@mayo.edu. Conflict of interest: The author has declared that no conflict of interest exists.

Nonstandard abbreviations used: Parkinson disease (PD); Lewy body (LB).
}

rites more widespread than previously recognized. For example, LBs are present in a high proportion of Alzheimer brains (8), and the basal ganglia contain many Lewy neurites (7). The report by Neumann et al. in this issue of the JCI moves the field forward another step (9). The authors have employed a method for detecting abnormal forms of $\alpha$-synuclein that may not necessarily be composed of fibrillar structures, which are the feature that permits ready detection of $\alpha$-synuclein in LBs and Lewy neurites in tissue sections.

\section{Histoblots detect \\ protease-resistant protein}

In this report (9) Neumann and coworkers applied a modification of a method, the histoblot (10), originally developed for detection of the pathologic form of prion protein (PrPres), which evidence suggests is the causative agent in transmissible spongiform encephalopathies such as CreutzfeldtJakob disease (11). The histoblot method takes advantage of the unusual solubility properties of $\mathrm{PrP}^{\mathrm{res}}$ (derived from the nonpathogenic cellular form of prion protein, $\mathrm{PrP}^{\mathrm{c}}$ that make it resistant to proteases. Sections of brain tissue are cut and applied to a nitrocellulose membrane support and subsequently treated with protease, which digests $\mathrm{PrP}^{c}$ and leaves an imprint of the distribution of PrPres in the remnants of tissue adherent to the nitrocellulose. The nitrocellulose is then immunostained much like a Western blot, but the result is an immunohistochemical imprint of the pathologic form of the protein freed from the staining of normal cellular protein, which is abundant for $\mathrm{PrP}^{\mathrm{c}}$. Instead of prion protein antibodies, Neumann and coworkers have used antibodies to $\alpha$-synuclein, and instead of transmissible spongiform encephalopathy tissue, they have used Lewy body disease and other disorders, such as multiple system atrophy, with abnormal $\alpha$-synuclein aggregates (Figure 1). As might be expected given the harsh treatment of the tissue, the method lacks fine resolution, but it does illustrate the general distribution of pathologic forms of $\alpha$-synuclein without the confound of normal cellular $\alpha$-synuclein, which is very abundant in synaptic termini in the neuropil of gray matter. The approach is not novel and has also been used to show abnormal forms of $\beta$-amyloid in human brains after ischemic stress (12). This is, however, the first use of a modification of histoblots to detect abnormal forms of $\alpha$-synuclein.

In addition to studies of human tissue, Neumann and coworkers also mapped $\alpha$-synuclein pathology in transgenic mice expressing human $\alpha$-synuclein (9). The method proved to be very sensitive and specific for illustrating the distribution of the abnormal form of $\alpha$-synuclein in the mouse brain, but the distribution does not map with the distribution of pathology in humans. A notable difference is that the midbrain tectum contained abundant protease-resistant $\alpha$-synuclein but the substantia nigra contained hardly any. In humans, the reverse would be expected. The problems with this transgenic model are not unique. Initial attempts to generate $\alpha$-synuclein transgenic mice that modeled PD met with limited success (13), but more recent models, including the one reported by Neumann and coworkers (9), have developed Lewy neurites and, occasionally, perikaryal inclusions that share some features with LBs from human brains $(14,15)$. Thus, $\alpha$-synuclein transgenic mice are models for $\alpha$-synuclein cytopathology, 


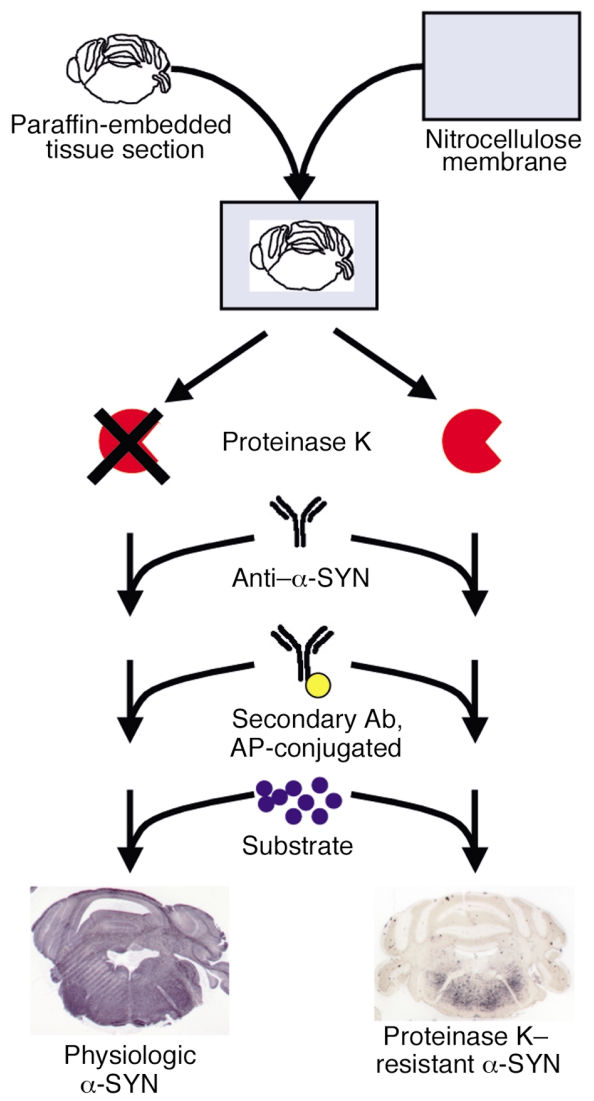

but not faithful models for the clinical disorder. Regions of the brain that are vulnerable to $\alpha$-synuclein-related pathology in transgenic mice overlap only loosely with human Lewy body disease, and behavioral abnormalities more often resemble motor neuron disease than they do an extrapyramidal disorder. Motor neurons are not vulnerable in human Lewy body disease.

\section{Antigen retrieval and abnormal forms of $\alpha$-synuclein}

Beyond demonstration of the distribution of pathologic forms of $\alpha$-synuclein, the study (9) confirms the results of previous studies that found altered forms of $\alpha$-synuclein in both animal models and humans. Previous studies have shown that $\alpha$-synuclein had abnormal solubility properties in Lewy body disease (16) and multiple system atrophy (17), but protease resistance had not been widely appreciated. Nevertheless, among the antigen retrieval methods that have been championed for demonstrating $\alpha$-synuclein pathology in brain tissue, protease treatment has been claimed to be among the most sensitive $(8,18)$. While it is possible that protease treatment reveals hidden epitopes in $\alpha$-synuclein, it is easier,

\section{Figure 1}

General principle of the PK-PET blot technique. Paraffin-embedded paraffin sections are blotted onto nitrocellulose membrane and dried. After deparaffinization and rehydration, the diagnostic blots are reacted harshly with the proteolytic enzyme PK (right), while control blots are incubated without PK (left). The enzymatic reaction is then quenched and followed by immunodetection of $\alpha$-synuclein using an appropriate primary antibody. Immunoreactive profiles are visualized by binding of a matched secondary antibody coupled to alkaline phosphatase (AP) which reacts with a colorigenic formazan dye. While undigested PET blots display the abundantly expressed "normal" $\alpha$-synuclein (lower left), the PK-PET blots reveal the pathological protease-resistant $\alpha$-synuclein (lower right). This procedure provides the basis of a novel diagnostic method to detect and characterize protein deposits in neurodegenerative diseases. Figure courtesy of Manuela Neumann and Philip Kahle. especially in light of the observations of Neumann et al. (9), to interpret the effects of protease treatment as enhanced detection of pathologic forms of $\alpha$-synuclein, because the normal cellular form is digested (Figure 2). The distinction between more widely used antigen retrieval methods and the histoblot method is that the histoblot may also detect a soluble, protease-resistant form of $\alpha$ synuclein that would be washed away in the traditional immunostaining approach. This issue might be addressed, but not completely resolved, if histoblots could be studied with electron microscopy to determine whether the protease-resistant $\alpha$-synuclein is nonfilamentous.

\section{Lewy body disease is a $\beta$-fibrillosis}

The biochemical basis for the protease resistance of $\alpha$-synuclein and $\operatorname{Pr}{ }^{\mathrm{res}}$ is not entirely known, but most current evidence suggests that protein conformation may play a significant role. Creutzfeldt-Jakob disease is the archetype of a neurologic disorder caused by an abnormal conformation of a normal cellular protein. Comparative investiga- tions of $\mathrm{PrP}^{\mathrm{res}}$ and $\mathrm{PrP}^{c}$ have shown that conformation rather than posttranslational modification is the best explanation for the differences (19). The abnormal conformer that is proteaseresistant has a high content of $\beta$-sheet secondary structure, and these properties fit well with the predicted properties of a "self-replicating" molecule (20). Fibrillar forms of $\alpha$-synuclein have also been shown to have high $\beta$-sheet content, while the soluble, nonfilamentous form of $\alpha$-synuclein is a natively unfolded protein with very little secondary structure (21). It is of more than passing interest that tau protein, the microtubule-associated protein that is the major structural component of neurofibrillary tangles in Alzheimer disease, shares a number of properties with $\alpha$-synuclein (22). Tau is also a natively unfolded protein with little secondary structure that forms pathologic filaments with unusual solubility properties and protease resistance. Unlike the fibrillar forms of synuclein, however, tau appears to contain little $\beta$ sheet secondary structure (23).

The characteristics of PrPres and protease-resistant $\alpha$-synuclein are also the characteristics of amyloid. Despite the

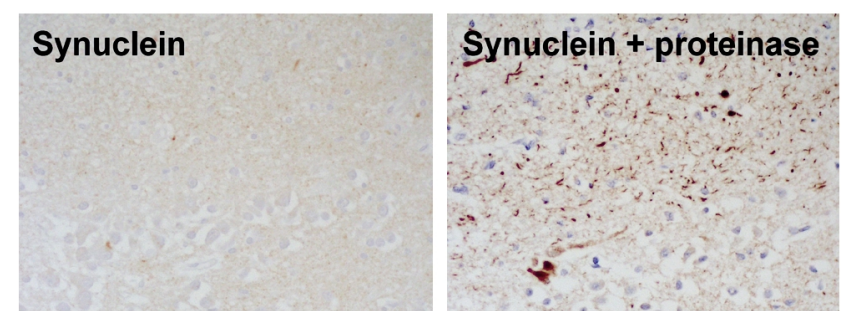

\section{Figure 2}

While Neumann and coworkers have demonstrated protease-resistant synuclein using a special technique, referred to as the histoblot (9), a similar phenomenon is also apparent with conventional immunohistochemistry, as demonstrated in two adjacent paraffin sections of the basal forebrain from a patient with Lewy body disease, immunostained for synuclein with no pretreatment or after pretreatment with proteinase $\mathrm{K}$. The protease-resistant synuclein, especially within neuritic processes, becomes evident after protease treatment, while the more diffuse punctate synaptic staining that represents normal synuclein is diminished by the treatment. 
wide clinical diversity and pathogenesis of amyloidoses, they share common properties, including increased frequency with age, defective proteolytic processing, and association with acidic macromolecules (24). The various amyloid molecules are structural variants of a normal precursor protein, and the major structural feature common to amyloid is $\beta$-sheet conformation, which led Glenner to refer to these disorders as the $\beta$-fibrilloses (25). There are many paths to amyloid, but in most cases the conversion of the normal precursor protein to the amyloid form of the protein involves overcoming a thermodynamic energy barrier. The size of the energy barrier and thus the likelihood of conversion of the normal to the pathologic form may be modified by a number of factors, such as posttranslational modification (e.g., proteolysis and phosphorylation) and association with chaperone proteins, lipids, divalent cations, or acidic macromolecules. A fundamental difference between prion diseases and the other $\beta$ fibrilloses that remains to be explained is that prion disease is an infectious or transmissible fibrillosis, while none of the other disorders has been shown to be transmissible.

1. Trétiakoff, C. 1919. Contribution à l'etude de l'anatomie pathologique du locus niger. Thesis. University of Paris. Paris, France.

2. Lewy, F.H. 1913. Zur pathologischen anatomie der paralysis agitans. Dtsch. Z. Nervenheilkd. 50:50-55.

3. McKeith, I.G., et al. 1996. Consensus guidelines for the clinical and pathologic diagnosis of dementia with Lewy bodies (DLB): report of the consortium on DLB international workshop. Neurology. 47:1113-1124.

4. Spillantini, M.G., et al. 1997. Alpha-synuclein in Lewy bodies. Nature. 388:839-840.

5. Kuzuhara, S., Mori, H., Izumiyama, N. Yoshimura, M., and Ihara, Y. 1988. Lewy bodies are ubiquitinated: a light and electron microscopic immunocytochemical study. Acta New ropathol. 75:345-353.

6. Dickson, D.W., et al. 1991. Hippocampal degeneration differentiates diffuse Lewy body disease (DLBD) from Alzheimer's disease: light and electron microscopic immunocytochemistry of CA2-3 neurites specific to DLBD. Neurology. 41:1402-1409.

7. Hamilton, R.L. 2000. Lewy bodies in Alzheimer's disease: a neuropathological review of 145 cases using alpha-synuclein immunohistochemistry. Brain Pathol. 10:378-384.

8. Duda, J.E., Giasson, B.I., Mabon, M.E., Lee, V.M., and Trojanowski, J.Q. 2002. Novel antibodies to synuclein show abundant striatal pathology in Lewy body diseases. Ann. Neurol. 52:205-210.

9. Neumann, M., et al. 2002. Misfolded proteinase $\mathrm{K}$-resistant hyperphosphorylated $\alpha$-synuclein in aged transgenic mice with locomotor deterioration and in human $\alpha$-synucleinopathies. J. Clin. Invest. 110:1429-1439. doi:10.1172/ JCI200215777.

10. Taraboulos, A., et al. 1992. Regional mapping of prion proteins in brain. Proc. Natl. Acad. Sci. USA 89:7620-7624.

11. Bockman, J.M., Kingsbury, D.T., McKinley, M.P. Bendheim, P.E., and Prusiner, S.B. 1985 Creutzfeldt-Jakob disease prion proteins in human brains. N. Engl. J. Med. 312:73-78.

12. Jendroska, K., et al. 1995. Ischemic stress induces deposition of amyloid beta immunoreactivity in human brain. Acta Neuropathol. 90:461-466.

13. Masliah, E., et al. 2000. Dopaminergic loss and inclusion body formation in alpha-synuclein mice: implications for neurodegenerative disor- ders. Science. 287:1265-1269.

14. Lee, M.K., et al. 2002. Human alpha-synucleinharboring familial Parkinson's disease-linked Ala-53-> Thr mutation causes neurodegenerative disease with alpha-synuclein aggregation in transgenic mice. Proc. Natl. Acad. Sci. USA. 99:8968-8973.

15. Giasson, B.I., et al. 2002. Neuronal alpha-synucleinopathy with severe movement disorder in mice expressing A53T human alpha-synuclein. Neuron. 34:521-533.

16. Baba, M., et al. 1998. Aggregation of alpha-synuclein in Lewy bodies of sporadic Parkinson's disease and dementia with Lewy bodies. Am. J. Pathol. 152:879-884.

17. Dickson, D.W., et al. 1999. Widespread alterations of alpha-synuclein in multiple system atrophy. Am. J. Pathol. 155:1241-1251.

18. Mori, F., Tanji, K., Yoshimoto, M., Takahashi, H., and Wakabayashi, K. 2002. Demonstration of alpha-synuclein immunoreactivity in neuronal and glial cytoplasm in normal human brain tissue using proteinase $\mathrm{K}$ and formic acid pretreatment. Exp. Neurol. 176:98-104.

19. Pan, K.M., et al. 1993. Conversion of alphahelices into beta-sheets features in the forma tion of the scrapie prion proteins. Proc. Natl. Acad. Sci. USA. 90:10962-10966.

20. Downing, D.T., and Lazo, N.D. 1999. Molecular modelling indicates that the pathological conformations of prion proteins might be beta-helical. Biochem. J. 343:453-460.

21. Conway, K.A., Harper, J.D., and Lansbury, P.T. Jr. 2000. Fibrils formed in vitro from alphasynuclein and two mutant forms linked to Parkinson's disease are typical amyloid. Biochemistry. 39:2552-2563.

22. Dickson, D.W. 1999. Tau and synuclein and their role in neuropathology. Brain Pathol. 9:657-661.

23. Sadqi, M., et al. 2002. Alpha-helix structure in Alzheimer's disease aggregates of tau-protein. Biochemistry. 41:7150-7155.

24. Sipe, J.D. 1992. Amyloidosis. Annu. Rev. Biochem. 61:947-975.

25. Glenner, G.G. 1980. Amyloid deposits and amyloidosis: the beta-fibrilloses. N. Engl. J. Med. 302:1283-1292 and 1333-1343. 